\title{
Formation continue sur mesure dans un cadre somptueux
}

\author{
Le $3^{\text {e }}$ Great Update de la Société Suisse de Médecine Interne Générale (SSMI) se \\ déroulera les 14 et 15 novembre 2013, pour la troisième fois dans le magnifique \\ Kursaal d'Interlaken. De nombreux intervenants offrent un programme passionnant \\ et orienté vers la pratique à l'attention des médecins de famille et des médecins hos- \\ pitaliers. Dans l'interview qui suit, les co-présidents de la manifestation apportent \\ des précisions sur le concept et le contenu de ces journées de formation continue.
}

\section{Lukas Zemp}

Sécretaire générale de la SSMI

Correspondance: Lukas Zemp Société Suisse de Médecine Interne Générale (SSMI) Case postale 422 CH-4008 Bâle Tél. 0612259335 sgim[at]sgim.ch

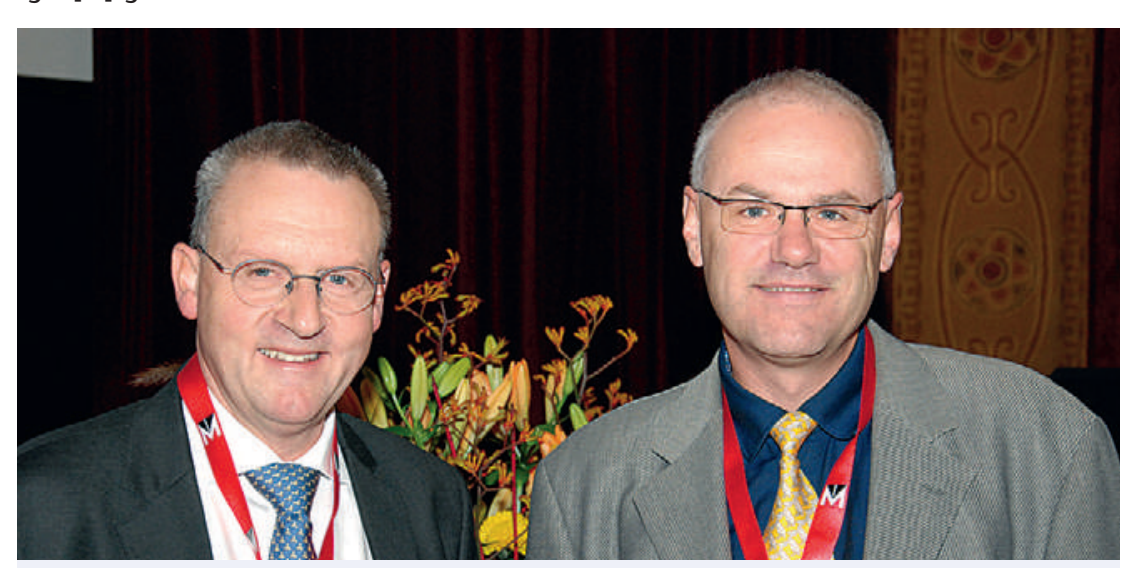

Les co-présidents du Great Update 2013 de la SSMI: Prof. Jörg D. Leuppi (à gauche) et Prof. Jonas Rutishauser.

Lukas Zemp: Qu'est-ce qui caractérise le Great Update de la SSMI? Quel est le lien avec l'assemblée annuelle de la SSMI?

Prof. Jonas Rutishauser: Le Great Update est un congrès exclusivement dédié à la formation continue et il s'adresse en premier lieu aux médecins de premier recours exerçant en cabinet. Toutefois, nos collègues praticiens hospitaliers qui envisagent d'exercer la médecine interne générale en cabinet tirent également profit des connaissances présentées. Avec le Great Update et l'assemblée annuelle, qui a également une orientation scientifique, la SSMI dispose d'une combinaison judicieuse de deux manifestations de formation continue attractives et complémentaires.

Jörg D. Leuppi: Grâce aux deux premières éditions, qui ont connu un vif succès, le Great Update s'est déjà solidement établi dans le programme de formation continue de la SSMI et il s'est aussi imposé auprès des formateurs, des exposants et des sponsors. Par ailleurs, nous nous sommes particulièrement réjouis de la forte participation de la Suisse romande lors des éditions de 2011 et 2012.
Le succès du Great Update de la SSMI était-il prévisible dès le départ? Quels retours avez-vous eu au sujet du Great Update 2012?

Jonas Rutishauser: Bien entendu, le succès n'était pas planifiable. Le Great Update a tout d'abord dû se forger son propre profil par rapport à l'assemblée annuelle de la SSMI, qui est la traditionnelle «réunion d'anciens élèves des internistes». Le Great Update, quant à lui, accorde davantage d'importance à la mise en œuvre pratique des nouvelles connaissances médicales.

Les retours au sujet du Great Update 2012 étaient à l'unanimité très positifs. Nous avons d'ailleurs tenu compte de diverses suggestions pour l'élaboration du programme de cette année, pour lequel nous sommes parvenus à obtenir la participation de nombreux intervenants du milieu hospitalier et ambulatoire. Ainsi, avec le Great Update 2013, nous sommes à nouveau en mesure de proposer un programme équilibré, qui répond de manière ciblée aux exigences des participants.

Leuppi: Avec l'aide d'un comité scientifique compétent et motivé et grâce au soutien d'un organisateur de congrès professionnel, l'IMK, nous sommes parvenus à imposer la nouvelle manifestation de formation continue de la SSMI au sein de l'environnement très compétitif des congrès et de la formation continue. Nous estimons qu'il est essentiel d'optimiser en permanence le programme du congrès et de répondre aux besoins des participants. C'est pourquoi la structure initiale, qui a entre-temps fait ses preuves, a été conservée: les ateliers, qui constituent le noyau central, sont accompagnés de conférences, de sorte que cette année à nouveau, les participants profitent d'un programme à la fois orienté vers la pratique et diversifié.

Quelle est la composition du comité scientifique du Great Update 2013?

Leuppi: Le comité scientifique du Great Update 2013 est composé de confrères praticiens et d'internistes 
hospitaliers: le comité compte deux co-présidents, qui sont le Prof. Jonas Rutishauser (Bruderholz) et moi-même (Prof. Jörg Leuppi, Liestal), ainsi que le Dr Romeo Providoli (Sierre), le Dr Sven Streit (Institut universitaire de médecine de famille, Berne) et le Dr Susanna Stöhr (Lucerne).

A quoi les participants doivent-ils exactement s'attendre lors du Great Update 2013 à Interlaken?

Leuppi: Le Great Update est une manifestation bilingue. Cette année également, des présentations en langues allemande et française se succèderont. Le programme inclut entre autres des conférences sur des thèmes comme la fibrillation auriculaire, les examens préopératoires, l'insuffisance cardiaque, les douleurs abdominales, les maladies de la thyroïde et le diagnostic des nodules pulmonaires.

\section{«Le Great Update accorde davantage d'importance}

\section{à la mise en œuvre pratique des nouvelles connaissan-}

\section{ces médicales.»}

le diagnostic de la dyspnée, la diabétologie avec des exemples de cas pratiques et l'interprétation de radiographies thoraciques. Les ateliers apportent également des conseils concernant des applications informatiques utiles pour la pratique et des précisions concernant la lecture critique d'articles scientifiques. Nouveauté de cette année: les «Hot Topics» présentés par les co-présidents de l'évènement.

\section{«Les ateliers, qui constituent le noyau central, sont accompagnés de conférences.»}

Pourquoi le Great Update 2013 se déroule-t-il à nouveau à Interlaken?

Leuppi: Le Great Update 2013 se tiendra à nouveau à Interlaken, qui a une situation géographique centrale et d'une grande beauté, car le centre des congrès Kursaal constitue un cadre parfait et personnalisé pour notre manifestation de formation continue. Les retours des participants nous l'ont confirmé.

Rutishauser: Comme l'année dernière, nous aurons de nombreuses opportunités de dialogue entre collègues du milieu ambulatoire et hospitalier. Et enfin, «last but not least», nous nous réjouissons de la soirée festive dans la très élégante brasserie de l'hôtel Victoria-Jungfrau à Interlaken! Vous êtes chaleureusement conviés à Interlaken.

Plus amples informations: informations sous http:// kongress2.imk.ch/SGIM2013/Home?language=fr ou www.sgim.ch
Rutishauser: Les ateliers sont animés par des internistes généralistes, par des collègues de disciplines spécifiques de médecine interne et par des représentants d'autres spécialités. Ces ateliers sont par exemple l'occasion d'évaluer des sédiments urinaires, de s'exercer au soin des plaies ou à la spirométrie ou d'acquérir des connaissances précieuses sur la réanimation. Parmi les autres thèmes des ateliers figurent

\section{Vous qui lisez une revue des Editions médicales suisses,}

\section{saviez-vous que ...}

- les Editions médicales suisses sont une coopération entre la Fédération des médecins suisses (FMH) et la plus ancienne maison d'édition et imprimerie au monde (les Editions Schwabe, fondées en 1488)?

- que les EMH sont les éditions de pointe en Suisse dans le domaine des journaux médicaux, avec dix revues spécialisées, des articles paraissant intégralement en ligne et un choix de livres de plus en plus large?

- que toutes les revues paraissant aux EMH sont les organes officiels de publication des sociétés médicales correspondantes?

Aux EMH, les articles sont publiés par des médecins pour des médecins. Des comités de rédaction indépendants constitués de spécialistes réputés assurent la qualité et l'actualité des articles qui couvrent toutes les disciplines médicales. Le choix des thèmes reflète les intérêts divers des médecins et des sociétés médicales et ne se concentre pas sur les aspects commerciaux.

Si vous souhaitez en savoir plus sur les EMH, vous trouverez plus d'informations sous www.emh.ch. Vous y trouverez également les revues publiées en ligne et des outils pratiques pour vos recherches d'articles. Nous sommes à votre disposition pour répondre personnellement à vos questions:

EMH Editions médicales suisses SA

Farnsburgerstrasse 8

$\mathrm{CH}-4132$ Muttenz
Marketing et communication

Tél. +41 (0)614678506/ Fax +41(0)614678556 marketing@emh.ch

EMH Editions médicales suisses - des publications à la pointe de la médecine 